\author{
J. LARSSON ${ }^{1,6, \infty}$ \\ A. ALLEN $^{2}$ \\ P.H. BUCKSBAUM ${ }^{5}$ \\ R.W. FALCONE ${ }^{4}$ \\ A. LINDENBERG ${ }^{4}$ \\ G. NAYLOR ${ }^{3}$ \\ T. MISSALLA ${ }^{1}$ \\ D.A. REIS 5 \\ K. SCHEIDT ${ }^{3}$ \\ A. SJÖGREN ${ }^{1}$ \\ P. SONDHAUSS ${ }^{2}$ \\ M. WULFF ${ }^{3}$ \\ J.S. WARK ${ }^{2}$
}

\section{Picosecond X-ray diffraction studies of laser-excited acoustic phonons in InSb}

\author{
${ }^{1}$ Atomic Physics Division, Lund Institute of Technology, P.O. Box 118, 22100 Lund, Sweden \\ ${ }^{2}$ Department of Physics, Clarendon Laboratory, University of Oxford, Parks Road, Oxford, OX1 3PU, UK \\ ${ }^{3}$ European Synchrotron Radiation Facility, Grenoble, 38043, France \\ ${ }^{4}$ Department of Physics, University of California, Berkeley, CA 94720, USA \\ ${ }^{5}$ Department of Physics, The University of Michigan, Ann Arbor, MI 48109, USA \\ ${ }^{6}$ Division of Synchrotron Radiation Physics, Lund University, P.O. Box 118, 22100 Lund, Sweden
}

Received: 22 March 2002/Revised version: 25 March 2002 Published online: 6 June 2002 • (C) Springer-Verlag 2002

ABSTRACT We have employed time-resolved X-ray diffraction with picosecond temporal resolution to measure the timedependent rocking curves of laser-irradiated asymmetrically cut single InSb crystals. Coherent acoustic phonons were excited in the crystals by irradiation with $800-\mathrm{nm}, 100$-fs laser pulses at irradiances between 0.25 and $12 \mathrm{~mJ} / \mathrm{cm}^{2}$. The induced time-dependent strain profiles (corresponding to the coherent phonons) were monitored by diffracting collimated, monochromatic pulses of X-rays from the irradiated crystals. Recording of the diffracted radiation with a fast low-jitter X-ray streak camera resulted in an overall temporal resolution of better than 2 ps. The strain associated with the coherent phonons modifies the rocking curve of the crystal in a time-dependent manner, and the rocking curve is recorded by keeping the angle of incidence of the X-rays upon the crystal fixed, but varying the energy of the incident X-rays around a central energy of $8.453 \mathrm{keV}$ (corresponding to the peak of the rocking curve of the unperturbed crystal). The observed time-dependent diffraction from the irradiated crystals is in reasonable agreement with simulations over a wide range of energies from the unperturbed rocking-curve peak.

PACS 63.20.Kr; 61.10.-i; 78.47.+p; 41.50.+h; 43.35.+d

1

\section{Introduction}

Over the past few years, significant progress has been made in the development of novel X-ray sources and detectors that provide temporal resolution in the picosecond or sub-picosecond domain. Several methods of such production and detection have been demonstrated. X-ray pulses of $K_{\alpha}$ radiation with durations of a few hundred femtoseconds can be produced by laser irradiation of materials at high $\left(\geq 10^{16} \mathrm{~W} / \mathrm{cm}^{2}\right)$ intensities $[1,2]$. Pulses of similar duration have been produced by Thomson scattering of femtosecond laser pulses from individual bunches of electrons within an accelerator. More recently it has been suggested by Schoenlein

E-mail: Jorgen.Larsson@fysik.Lth.se et al. [3] that such X-ray pulses may also be produced by chopping a femtosecond section of electrons out of the standard electron bunch in a third-generation synchrotron. Evidence demonstrating the feasibility of this novel idea has been obtained in the infrared region of the spectrum. In addition to the methods cited above, it is also possible to obtain picosecond resolution at present third-generation synchrotron sources by employing X-ray streak cameras that are synchronized to better than a picosecond with the laser that pumps the sample. The laser in turn is synchronized to the electron bunches circulating within the synchrotron to about 10 ps. This method, which we employ in the work presented here and describe in more detail in Sect. 3 has now been implemented at a number of synchrotron sources.

The availability of such temporal resolution in the X-ray region of the spectrum has led to several novel time-resolved $\mathrm{X}$-ray-diffraction experiments. In particular, the diffraction of X-rays from crystals within which coherent phonons have been generated by irradiation with a femtosecond time scale laser pulse has attracted considerable interest. In these experiments, a single-crystal semiconductor is irradiated with a laser pulse with a pulse length of the order of $100 \mathrm{fs}$. The laser energy is absorbed by the electrons, exciting them across the band gap, and further heating them. The electron energy is rapidly transferred to the lattice via the generation of longitudinal optical phonons, and finally acoustic phonons, on time scales that are typically of the order of a few picoseconds. The resultant heating of the lattice is thus generally so rapid that it occurs isochorically - i.e. the penetration depth of the heating laser is such that the lattice heating is rapid compared with the penetration depth divided by the sound speed within the crystal. The resultant stress within the crystal is then relieved by expansion of the crystal, starting from the surface. By conservation of momentum, a compression wave is also launched into the crystal. Analytic solutions for this resultant strain profile exist under the assumption of instantaneous heating of the lattice, an exponential decay in initial stress (due to the exponential fall-off in absorbed laser energy), and neglecting phonon dispersion. This time-dependent strain profile can be thought of as a spectrum of coherent phonons, with wave vectors approximately centered about the inverse 
of the penetration depth of the heating laser beam. Such picosecond ultrasonic pulses have been studied for many years in a variety of materials by observing the resultant changes in the optical properties (e.g. reflectivity) of the crystal due to the propagating strain profile - for example see the review article by Maris [4]. Picosecond X-ray diffraction offers a complementary method to observe such coherent phonons, and may prove to have significant advantages over optical techniques in terms of a more direct method for deducing the strain profiles present within the irradiated crystals, as well as being useful for studying such strain pulses in opaque crystals. The first experiments in this area were performed with laser-plasma-generated $K_{\alpha}$ radiation as the X-ray probe [2]. Whilst gross features in the diffraction pattern corresponding to the strained lattice were observed, the finite bandwidth and relatively low intensity of the source prevented the direct observation of the oscillations in the diffracted X-rays that are associated with diffraction from high-frequency phonons. In contrast, experiments performed with the Advanced Light Source (ALS) synchrotron at the Lawrence Berkeley Laboratory produced direct evidence of such effects. Furthermore, in these experiments it was deduced that good agreement between the observed and computed rocking curves could only be found if a finite electron-phonon coupling time was invoked, resulting in a smoother transition from regions of expansion and compression within the strained crystal.

In this paper we present results of picosecond X-raydiffraction studies of coherent phonons in InSb. The experiments were performed at the European Radiation Synchrotron Facility, and differ from the above-mentioned ALS studies in several ways. Firstly, the energy of the diffracting X-rays was considerably higher - centered around $8.453 \mathrm{keV}$ (in contrast to $4.8 \mathrm{keV}$ used in the ALS experiments). Secondly, and as discussed in more detail below, the diffraction geometry employed was markedly different, resulting in a significant reduction in the effects of the finite bandwidth of the source, and allowing a clearer observation of the oscillations that the coherent phonons impose upon the diffracted X-rays. Thirdly, the loss of oscillations seen in the ALS experiment was at the damage point. In this paper we perform the experiment at much lower fluence and demonstrate that the only dephasing seen under these conditions are due to the finite bandwidth. Finally, data on the time-dependent reflectivity of the crystal was collected over a large number and wide range of X-ray energies away from the central energy (which corresponded to the peak of the rocking curve for an unperturbed crystal). Such a range of data provides the opportunity for detailed comparison with simulations.

The paper is laid out in the following manner. Before discussing the experiments, we outline the form of the results expected by considering the theory of dynamical diffraction from high-frequency coherent phonons propagating along the surface normal of a perfect crystal. Diffraction from asymmetrically cut crystals (where the reciprocal lattice vector of the diffracting plane is not parallel to the surface normal of the crystal) is discussed - in particular the advantages of using an asymmetrically cut crystal such that the X-rays are incident upon the surface of the crystal at grazing angles. The experimental results are then presented, before a comparison between them and the simulations based on dynamical diffraction theory. We conclude with a discussion of the results, and ideas for further work.

\section{$2 \quad X$-ray diffraction from coherent phonons}

Coherent phonons, be they acoustic or optical, introduce an additional periodicity into the crystal. From this we deduce that we might expect to observe diffracted radiation at angles other than the original Bragg angle simply from wave-vector-matching considerations. Figure 1a illustrates in schematic form the diffraction of an incident X-ray of wave vector $\boldsymbol{k}_{0}$ into a wave of wave vector $\boldsymbol{k}^{\prime}$ from a plane with reciprocal lattice vector $\boldsymbol{G}_{\mathrm{H}}$. The index $\mathrm{H}$ denote lattice points $(h k l)$ in reciprocal space. The normal Bragg condition is given by $2 \boldsymbol{k}_{0} \cdot \boldsymbol{G}_{\mathrm{H}}=\left|\boldsymbol{G}_{\mathrm{H}}\right|^{2}$, i.e. $\boldsymbol{k}_{0}+\boldsymbol{G}_{\mathrm{H}}=\boldsymbol{k}^{\prime}$. However, if we assume coherent phonons of wave vector $\boldsymbol{q}$ exist within the crystal, then we might expect the Bragg condition to be met when $\boldsymbol{k}_{0}+$ $\boldsymbol{G}_{\mathrm{H}} \pm \boldsymbol{q}=\boldsymbol{k}^{\prime}$ as shown in Fig. 1b. Thus diffraction will occur at angles other than the original Bragg angle: by geometry we find that diffraction will occur at an angle $\Delta \theta$ from the Bragg angle for the unperturbed crystal, $\theta_{\mathrm{B}}$, such that

$\Delta \theta= \pm \frac{|\boldsymbol{q}|\left(\tan \theta_{\mathrm{B}} \cos \phi+\sin \phi\right)}{\left|\boldsymbol{G}_{\mathrm{H}}\right|}$,

where $\phi$ is the asymmetry angle (the angle between the surface normal and $\boldsymbol{G}_{\mathrm{H}}$ ). The asymmetry angle $\phi$ is taken to be positive when the angle between the incident X-rays and the crystal surface is smaller than $\theta_{\mathrm{B}}$, and negative when the angle is larger than $\theta_{\mathrm{B}}$. Thus if the speed of sound within the crystal is $v$, we would expect an oscillatory signal in the timeresolved diffraction with an angular frequency $\omega$ given by

$\omega \approx v \Delta \theta \frac{\left|\boldsymbol{G}_{\mathrm{H}}\right|}{\left(\tan \theta_{\mathrm{B}} \cos \phi+\sin \phi\right)}$.

These simple wave-vector-matching considerations suggest that oscillatory diffraction will be observed, but provide
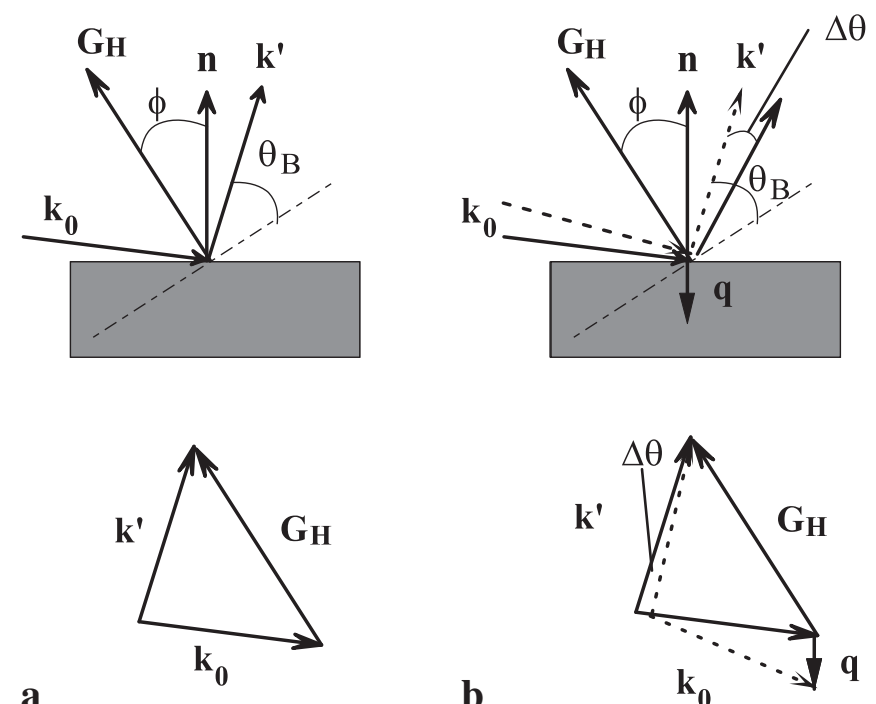

a

b

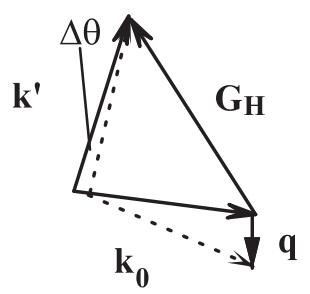

FIGURE 1 Diffraction of X-rays of wave vector $\boldsymbol{k}_{0}$ from planes with reciprocal lattice vectors $\boldsymbol{G}_{\mathrm{H}}$ in $\mathbf{a}$ the absence and $\mathbf{b}$ the presence of phonons of wave vector $\boldsymbol{q}$. The relative lengths of $\boldsymbol{q}, \boldsymbol{G}_{\mathrm{H}}$ and the $\boldsymbol{k}$-vectors are not to scale. The dotted lines are the incident and diffracted $\boldsymbol{k}$-vectors in the presence of the pure phonon 
no information about the reflected intensities, or the form of the diffraction for a spectrum of coherent phonons, as is excited by short-pulse laser irradiation of a crystal. For a more detailed treatment of such effects, we employ dynamical diffraction theory.

\subsection{X-ray diffraction from coherent phonons: dynamical diffraction theory}

The modification of dynamical diffraction theory to incorporate the effects of strain within crystals has been discussed by many authors, starting with the independent work of Takagi [5] and Burgeat and Taupin [6]. Within this theory the susceptibility of the lattice is taken to be periodic, i.e. can be expanded into a Fourier series with Miller indices $\mathrm{H}=h k l$ and the complex Fourier coefficients $\psi_{\mathrm{H}}$.

$\psi=\sum_{\mathrm{H}} \psi_{\mathrm{H}} \mathrm{e}^{-\mathrm{i} \boldsymbol{G}_{\mathrm{H}} \cdot \boldsymbol{r}}$

The complex wave amplitudes of the incident $\mathrm{X}$-rays, $D_{0}$, and diffracted X-rays, $D_{\mathrm{H}}$, are linked by the coupled differential equations

$\mathrm{i} \frac{\lambda \partial D_{0}}{\pi \partial x_{0}}=\psi_{0} D_{0}+\psi_{\overline{\mathrm{H}}} D_{\mathrm{H}}$

$\mathrm{i} \frac{\lambda \partial D_{\mathrm{H}}}{\pi \partial x_{\mathrm{H}}}=\psi_{0} D_{\mathrm{H}}+\psi_{\mathrm{H}} D_{0}-\alpha_{\mathrm{H}} D_{\mathrm{H}}$,

where $x_{0}$ is the distance along the direction of the incident ray and $x_{\mathrm{H}}$ the distance along that of the diffracted ray. The quantity $\alpha_{\mathrm{H}}$ is associated with the deviation from the Bragg angle and, for a perfect crystal, is given by

$\alpha_{\mathrm{H}}=\frac{\boldsymbol{G}_{\mathrm{H}}^{2}+2 \boldsymbol{G}_{\mathrm{H}} \cdot \boldsymbol{k}_{0}}{\boldsymbol{k}_{0}^{2}} \equiv-2\left(\theta-\theta_{\mathrm{B}}\right) \sin \left(2 \theta_{\mathrm{B}}\right)$.

Longitudinal coherent phonons can be incorporated into the dynamical theory by noting that they alter the local Bragg angle owing to the associated straining of the lattice. If we introduce a local one-dimensional strain $\eta(r)$ into the crystal (parallel to the surface normal), then the parameter $\alpha_{\mathrm{H}}(r)$ is given by

$\alpha_{\mathrm{H}}(r)=-2(\Delta \theta-C \eta(r)) \sin \left(2 \theta_{\mathrm{B}}\right)$,

with

$C=\cos ^{2} \phi \tan \theta_{\mathrm{B}}+\sin \phi \cos \phi$.

For a given strain profile within the crystal, and a given Bragg angle, a solution of (4) and (5) will yield the ratio $\left|D_{\mathrm{H}} / D_{0}\right|^{2}$ at the surface of the crystal - i.e. the X-ray reflectivity. We obtain such a solution computationally by the method put forward by Wie et al. [7]. In order to model timedependent diffraction, we solve the equations for the instantaneous strain at each given time (the rate of change of strain is slow compared with the time taken for the $\mathrm{X}$-rays to traverse an extinction depth. Thus the explicit time dependence of the wave fields within the crystal, as discussed by Wark and Lee [8], need not be taken into account here).
In order to illustrate the above discussion, in Fig. 2 we show a simulation of the time-dependent diffraction of $10-\mathrm{keV}$ $\mathrm{X}$-rays from a single-mode coherent phonon of peak strain amplitude $\pm 10^{-4}$ and wavelength $200 \mathrm{~nm}$ propagating along the surface normal in a GaAs(111) crystal. The diagram shows the logarithm of the reflectivity as a function of time and angular deviation from the Bragg angle, with the phonons being introduced into the crystal at $t=0$. Note the slight offset of the main Bragg peak from the Bragg angle due to the mean refractive index of the crystal. Either side of the reflection curve, at \pm 65 arcsec from the main Bragg peak, we see the weaker sidebands corresponding to the diffraction from the coherent phonons - this being the angular deviation predicted by (1). These sidebands clearly exhibit a temporal oscillation, as predicted by (2).

However, in actual experiments involving such highfrequency $(0.1 \mathrm{THz})$ acoustic phonons, a broad spectrum of coherent phonons, rather than a single mode, is generated [2,9-11]. This spectrum of phonons is introduced into the crystal by irradiation with a femtosecond time-scale laser pulse, resulting in a complicated strain profile propagating into the bulk of the crystal.

When the semiconductor crystal is irradiated, the laser energy is absorbed by the electrons, which are excited across the band gap and heated. In the limit of instantaneous transfer of energy from the electrons to the acoustic phonons the lattice is heated at constant volume, producing a stress profile that decays exponentially within the crystal (assuming exponential absorption of the laser energy, and linear thermal expansion). Subsequently the surface of the crystal starts
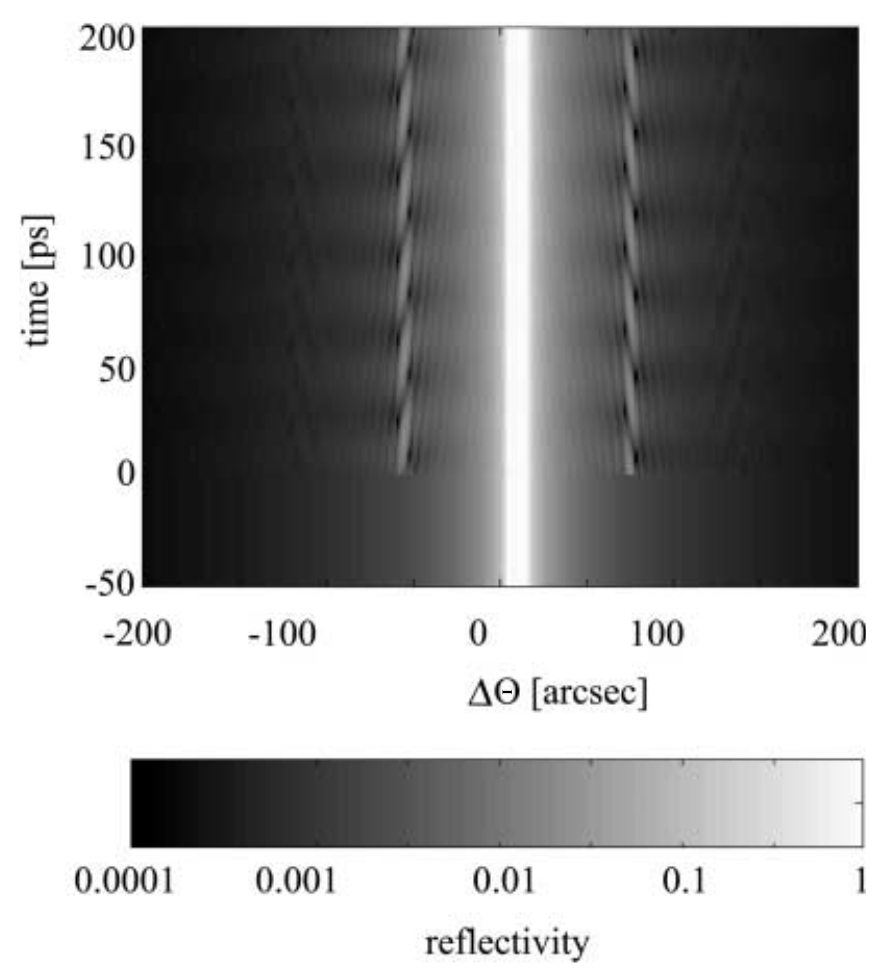

FIGURE 2 Simulation of the diffraction of 10-keV X-rays from the (111) plane of a GaAs symmetrically cut crystal within which pure acoustic phonons of wavelength $200 \mathrm{~nm}$ are propagating along the direction of the surface normal. The peak strain induced by the phonons is assumed to be $\pm 10^{-4}$ 

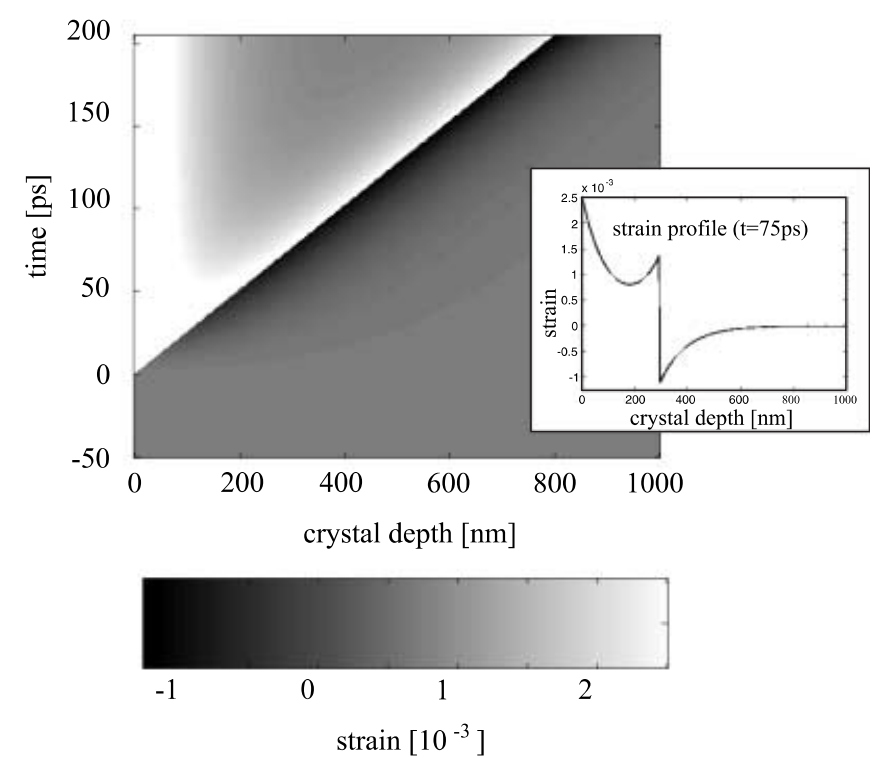

FIGURE 3 Computed strain as a function of depth within the InSb crystal assuming instantaneous heating with a $1 /$ e depth of $100 \mathrm{~nm}$. The lattice temperature at the crystal surface is assumed to be just below the melting point. Diffusion, finite electron-phonon coupling time and phonon dispersion have been ignored. The inset shows a line out of the strain-depth profile taken at $75 \mathrm{ps}$

to expand and by conservation of momentum a compression wave is launched into the bulk of the crystal. In the experiments we shall describe, the diameter of the laser spot heating the crystal is very large compared with the laser penetration depth, and thus the strain pulse launched into the crystal is one-dimensional, and strain occurs only along the surface normal. A time-dependent solution to the strain-depth profile (ignoring phonon dispersion) has been found by Thomsen et al. for such conditions [12]. A typical strain-depth profile calculated under these assumptions for the irradiated InSb is shown in Fig. 3, where we have assumed that the laser beam is absorbed with a $1 /$ e depth of $100 \mathrm{~nm}$, and the laser intensity is such that the surface of the crystal is just below the melting point. We note that this strain profile is effectively a superposition of coherent phonons, with wave vectors peaked around an inverse of the laser penetration depth.

\subsection{X-ray diffraction from coherent phonons: geometry and bandwidth}

We have noted that a femtosecond laser will introduce a time-dependent strain profile into the crystal, corresponding to a spectrum of coherent phonons. However, the detailed corresponding time-dependent X-ray reflectivity as a function of angle will depend on several factors: most notably the X-ray energy, the experimental geometry and the bandwidth of the source. In this subsection we illustrate the importance of the effects of geometry and finite bandwidth by simulating time-dependent rocking curves corresponding to the strain profiles given in Fig. 3 for two different cases. These cases correspond to the experimental conditions used in the work of Lindenberg et al. at the ALS, and to our own experimental work that will be discussed in Sect. 4, and will illustrate the motivation behind our particular choice of experimental geometry.
The absolute value of the absorption length of X-rays is typically long compared with the laser penetration depths. Thus, in order to maximize the reflectivity from the coherent phonons it has been suggested that asymmetric diffraction should be used - that is to say that the crystal is cut such that the reciprocal lattice vector of interest makes an angle to the surface normal. In this case, the X-rays are incident, or are diffracted (depending on the orientation of $\boldsymbol{G}_{\mathrm{H}}$ with respect to the surface normal and incident X-rays - i.e. the sign of $\phi$ ), at an angle with respect to the crystal surface which is less than the Bragg angle. This angle can be made grazing, better matching the component of the laser and X-ray penetration depths along the surface normal (note that within the crystal the laser propagates in a direction very close to the opposite of the surface normal even for grazing incidence due to the high optical refractive index of the sample, and thus refraction at the sample surface). Given that any source has a finite bandwidth, we show below that the choice of the sign and magnitude of $\phi$ can have significant implications for the observation of the coherent phonons within the X-ray signal.

In the work of Lindenberg et al., $\phi$ was chosen to be negative, and thus the X-rays were diffracted at grazing incidence. In their experiments, X-rays of energy $4.8 \mathrm{keV}$ and spectral bandwidth $4 \mathrm{eV}$ were diffracted from the (111) planes of an irradiated InSb crystal, and recorded on a streak camera. The crystals were asymmetrically cut and aligned such that $\phi=-3^{\circ}$.

Figure 4 shows the computed logarithm of the timedependent reflectivity assuming the strain profile given in Fig. 3. Note that, if data is collected at a particular angular position away from the original Bragg angle, temporal oscillations will be observed in the X-ray reflectivity as in the single-phonon case. This simulation has assumed perfectly monochromatic X-rays. In order to take into account the bandwidth of the X-ray source, we note that a finite bandwidth in energy corresponds to an effective angular width given by

$\Delta \theta_{\mathrm{bw}}=\frac{\Delta E}{E} \tan \theta$.

Thus in Fig. 5 the bandwidth effects have been simulated in an approximate manner by convolving Fig. 4 with a Gaussian function of FWHM $\Delta \theta_{\mathrm{bw}}$. Note that this reduces the visibility of the temporal oscillations.

In contrast to the experiments of Lindenberg et al., in the work described below we used an experimental geometry such that $\phi$ was positive. The potential advantages of such a geometry may be seen by a consideration of (1), (7) and (8). If the angular resolution of the experiment is limited by the effective bandwidth, as in (9), then it is good practice to make the dispersion of the system as high as possible. This occurs in the positive- $\phi$ geometry, with a larger angular deviation for a given value of strain and phonon wave vector. If the crystal were uniformly strained, a larger angular deviation would occur: this can be seen by considering the factor $C$ of (7) and (8). With $\phi$ positive, $C$ is large, as we need to add rather than subtract the two terms in (8). The underlying physics of this increase in dispersion for a given strain is that when we introduce a one-dimensional strain into the crystal the planes not only change in spacing, but they also rotate. If the X-rays are incident at grazing angles these two effects combine, whereas 

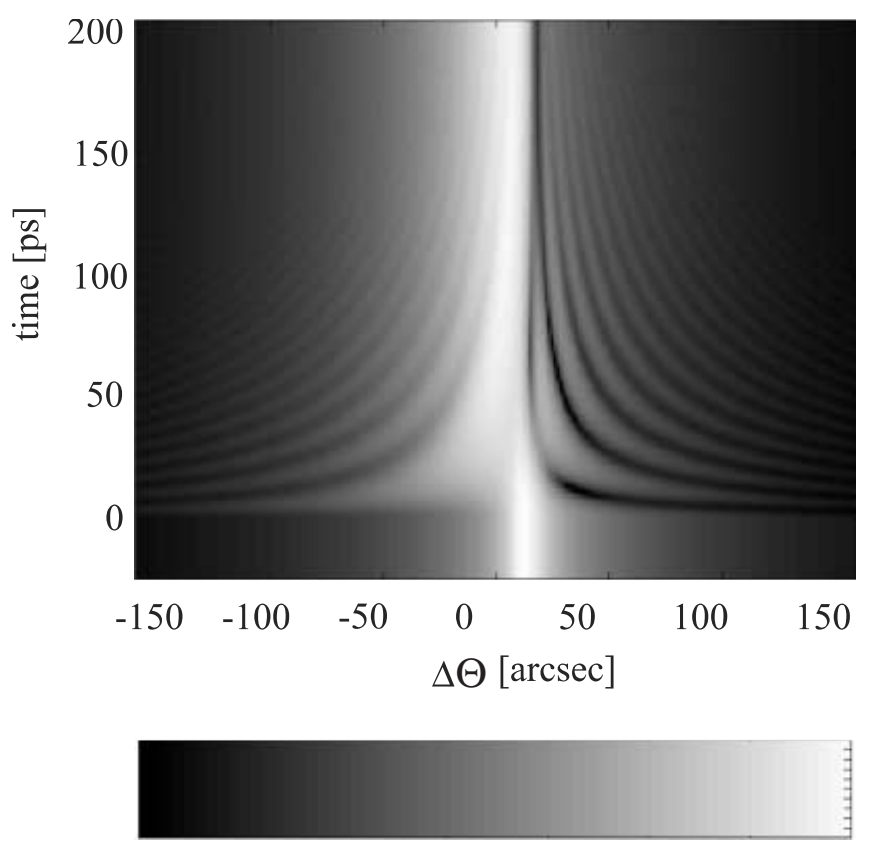
0.0001
0.001
0.01
reflectivity

0.1

FIGURE 4 Simulated time-dependent X-ray diffraction for 2.4- $\AA$ purely monochromatic radiation from the asymmetric InSb crystal described in the text, assuming the strain history plotted in Fig. 3

if the X-rays leave at grazing incidence they tend to cancel out. In the latter case, the rocking curve of the sample crystal becomes narrow, and there is only a small angular deflection for a given value of strain within the crystal. Similarly, for oscillatory strains (i.e. coherent acoustic phonons), a given phonon wave vector gives rise to a larger angular deviation for positive $\phi$, as can be seen from (1).

Furthermore, it is clear that the bandwidth of the incident radiation should be as low as possible. To this end the channelcut crystal monochromator, usually employed in synchrotron X-ray sources, should ideally be operated with its diffracting planes parallel to those of the sample crystal. Under such circumstances, if the interplanar spacings of the crystal monochromator and the sample crystal match well, the effective bandwidth is just limited by the rocking-curve width of the monochromator. Unfortunately, for the experiments we describe here, the relative positions of the monochromator and the streak camera were such that such a parallel arrangement was not possible, and the effective bandwidth of the source was $2 \mathrm{eV}$, with a mean energy of $8.453 \mathrm{keV}$. The InSb (111) crystal was cut such that $\phi=7.2^{\circ}$. In Fig. 6 we show the predicted time-dependent rocking curve (i.e. for monochromatic radiation) for these parameters, once again assuming the strain profile shown in Fig. 3. It can be seen that the angular dispersion is now considerably higher, due to the use of the positive- $\phi$ geometry. As the dispersion is so high, the effect of the finite bandwidth of the source is not so detrimental, as can be seen in Fig. 7, and the visibility of the fringes is considerably improved. Using a positive- $\phi$ geometry increases the dispersion, but introduces the associated problem that the footprint of the X-ray beam on the crystal surface is increased. Thus the footprint of the laser irradiating the
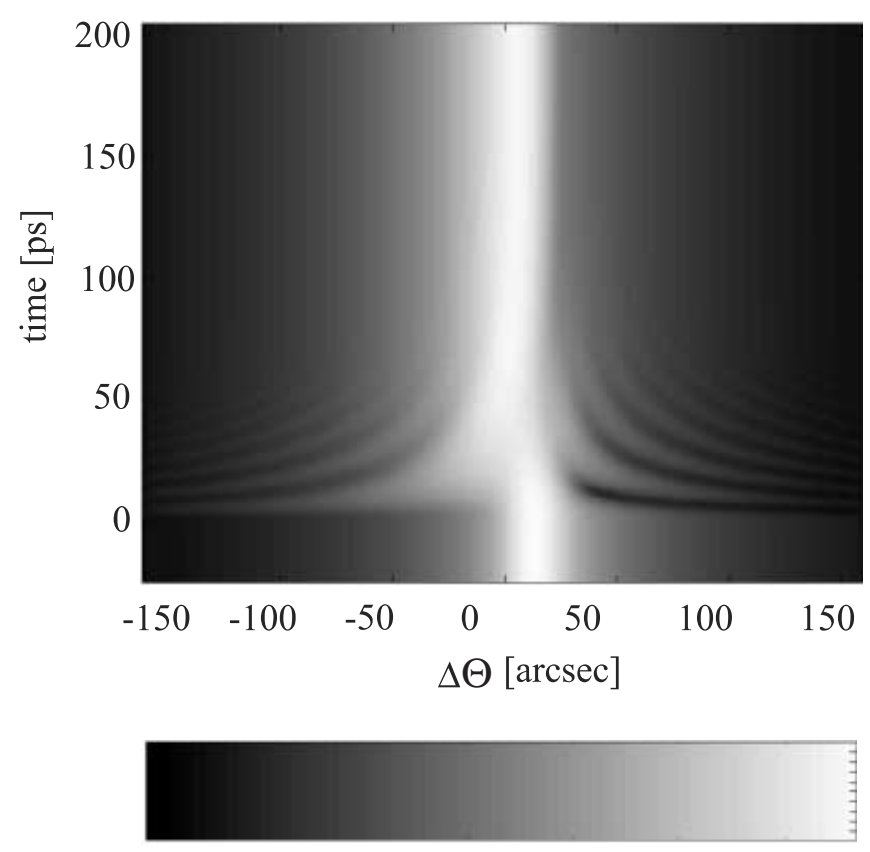

$$
\begin{array}{llr}
0.0001 & 0.001 & 0.01 \\
& & \text { reflectivity }
\end{array}
$$

FIGURE 5 Simulated time-dependent X-ray diffraction for $2.4-\AA \AA$ radiation from the asymmetric (negative- $\phi$ ) InSb crystal described in the text, assuming the strain history plotted in Fig. 3, and taking into account the finite bandwidth of the $\mathrm{Si}$ (111) monochromator used (FWHM = $11 \operatorname{arcsec})$
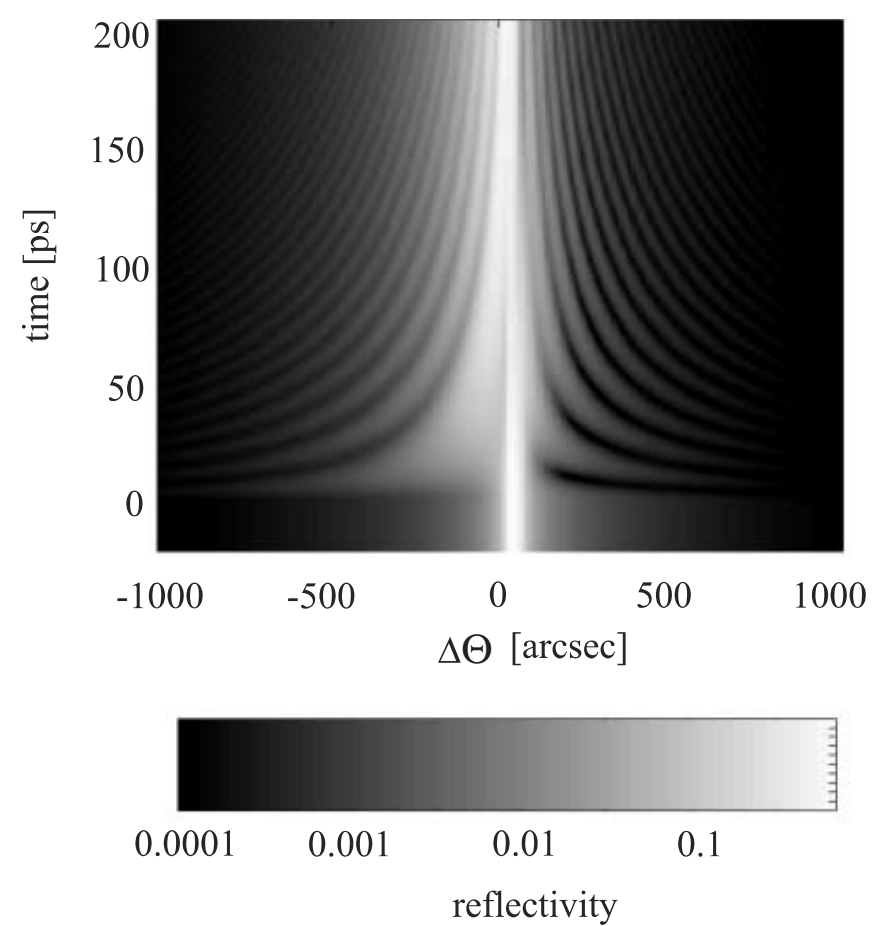

FIGURE 6 Simulated time-dependent Bragg diffraction of a $8.453-\mathrm{keV}$ monochromatic X-ray beam from the asymmetrically cut InSb with positive $\phi\left(=7.2^{\circ}\right)$

crystal (which should be as co-linear with the X-ray beam as possible to minimize the temporal smearing) must also be increased, and thus higher laser energies are required for a given irradiance. 

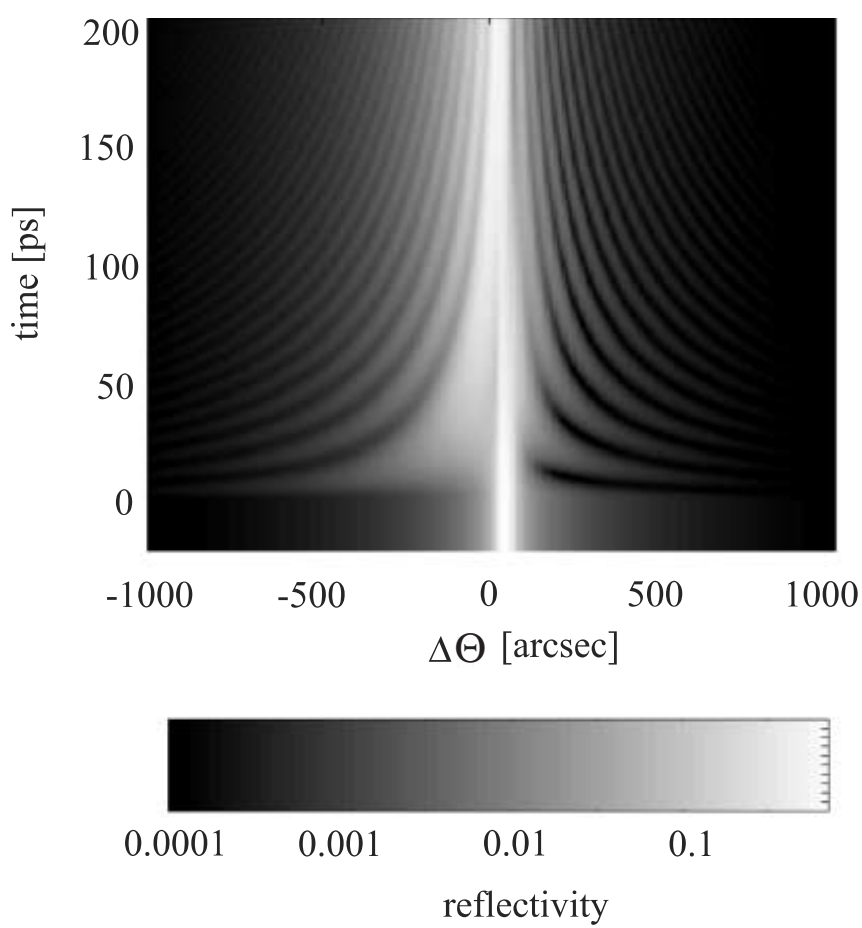

FIGURE 7 Simulated time-dependent Bragg diffraction of a 8.453-keV monochromatic X-ray beam from the asymmetrically cut InSb with positive $\phi=7.2^{\circ}$ ), but taking into account the experimentally determined bandwidth of the radiation, which corresponds to an angular width of $\mathrm{FWHM}=8 \operatorname{arcsec}$

Having given a description of the methods used to simulate picosecond X-ray diffraction from coherent phonons, and explained the motivation behind using asymmetrically cut crystals and a positive- $\phi$ geometry, in Sect. 3 we give a description of the experimental setup, before presenting the experimental results, and comparing them with simulations.

\section{3}

\section{Experiments}

The experiments were performed at the beamline ID9 of the European Synchrotron Radiation Facility (ESRF). The instrumentation at this beamline has been developed to provide capabilities for time-resolved X-ray experiments with picosecond time resolution. A laser with femtosecond pulse duration, synchronized to the X-ray radiation from the synchrotron, allows two distinct types of experiments. Firstly, pump-probe experiments can be performed with a temporal resolution limited by the duration of the X-ray pulses $(\approx 100 \mathrm{ps})$. Secondly, an ultra-fast X-ray streak camera, synchronized with the laser, makes studies with a temporal resolution in the 1-ps range possible. In this study the majority of the results presented were obtained using the second method.

A Ti: $\mathrm{Al}_{2} \mathrm{O}_{3}$-based, $100-\mathrm{fs}, 900-\mathrm{Hz}$ repetition rate laser was synchronized to the radio-frequency clock (and thus the electron bunches) of the ESRF electron-storage ring with a jitter less than $10 \mathrm{ps}$. X-rays were produced as the electron bunches passed through the U20 undulator. The undulator beamline (ID9) provides tunable X-rays using a twocrystal monochromator, and the mean X-ray energy was set at $8.453 \mathrm{keV}$. The X-rays were focused using a toroidal mirror yielding an X-ray spot size of approximately $0.1 \mathrm{~mm}^{2}$ in the plane of the sample. A mechanical chopper, again syn- chronized with both the laser clock and the clock of the synchrotron [13], was used to reduce the pulse repetition rate of the X-rays to $900 \mathrm{~Hz}$ in order to match the laser repetition rate. The divergence of the X-ray beam was approximately $10 \mu \mathrm{rad}$. The temporal delay between the laser and the 100-ps $\mathrm{X}$-ray pulse could be varied using an electronic phase shifter in the rf signal used to synchronize the laser oscillator. The experimental setup is shown in schematic form in Fig. 8.

In order to obtain a temporal resolution better than that dictated by the X-ray pulse duration (and, indeed, than the jitter between the laser pulses and the synchrotron) an X-ray streak camera equipped with a CsI transmission photocathode was used [14]. The streak camera was also operated at the repetition rate of $900 \mathrm{~Hz}$, with a time resolution of $500 \mathrm{fs}$. It is important to stress that the overall temporal resolution is determined by the jitter between the streak camera and the laser, and not that between the laser and the electron bunches within the synchrotron. This is due to the fact that the laser is used both to initiate the event of interest in the sample (i.e. generate the coherent phonons) and to trigger the sweep voltage to the deflection plates of the streak camera with a lasertriggered photoconductive switch [15]. Thus, although the 100 -ps X-ray pulse has a jitter of the order of 10 ps with respect to the laser, the position on the streak camera output screen corresponding to a particular event within the crystal only varies by the jitter between the laser and the streak camera. Indeed, in collecting the data (often averaged over tens of thousands of shots, as will be described in more detail below), the delay between the laser and X-rays was deliberately slowly varied during the data-collection process, by using the electronic phase shifter referred to above. In this manner, in one data-collection run it was possible to record the reflectivity of the perturbed crystal over a time period longer than that of the individual X-ray pulses. The slow variation of the time recording of the X-ray pulse, as multiple pulses were collected, typically resulted in an averaged $\mathrm{X}$-ray pulse with an approximately top-hat temporal profile and an effective duration several times that of the intrinsic synchrotron bunch length. The collection and averaging of data over multiple pulses also helped to increase the signal-tonoise ratio.

A beam splitter placed in the laser beam produced two pulses, one of which was used to irradiate the sample of interest, and the other to synchronize the streak camera. Approximately $100-\mu \mathrm{J}$ of $800-\mathrm{nm}$ light from the $\mathrm{Ti}: \mathrm{Al}_{2} \mathrm{O}_{3}$ laser was used to irradiate the GaAs photoconductive switch which triggered the streak camera. UV light, with a wavelength of $266 \mathrm{~nm}$, was then obtained by frequency mixing the 800-nm light in two KDP crystals. This UV radiation was then incident through the side of the streak camera front end onto a region of the photocathode separated from that where the $\mathrm{X}$-rays were recorded. This UV pulse was then used to calibrate the time response and relative jitter of the streak camera with respect to the laser. By recording this UV signal over the exact same laser pulses as those used for X-ray data collection, the effective temporal resolution of the streak-camera system was found to be $4 \mathrm{ps}$ (rms) in this averaging mode. The output phosphor screen of the streak camera was fiberoptically coupled by an image intensifier, which in turn was fiber-coupled to a slow-scan CCD camera. The output of the 


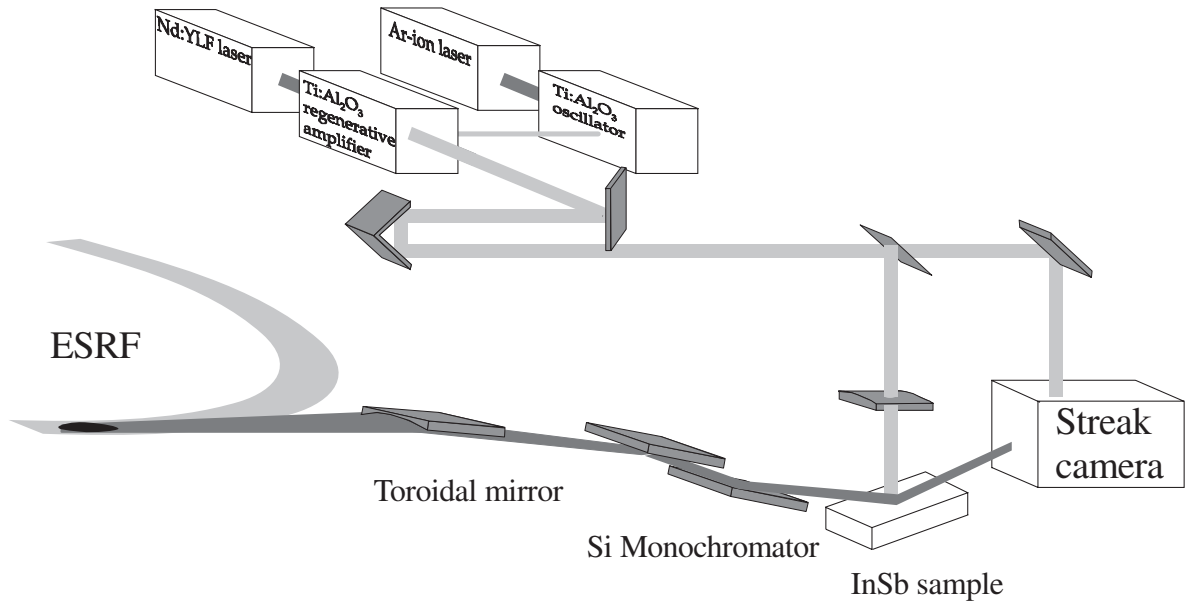

FIGURE 8 A schematic diagram of the experimental setup
CCD was read out at the end of the registration of multiple $\mathrm{X}$-ray and laser pulses.

The main portion of the laser beam was used to irradiate the InSb wafer to produce the coherent phonons while the $\mathrm{X}$-rays diffracted from the crystal. The optical path of this laser beam was constructed so that its path of incidence onto the crystal was as close as possible to being co-linear with the X-rays. In order to determine the absolute temporal position on the streak-camera output corresponding to the time when the laser irradiated the crystal, the following procedure was adopted. Firstly, the beryllium window in front of the CsI photocathode which was in place during the X-ray data collection was replaced with a fused-silica window. A frequencydoubling crystal was then inserted into the main laser beam. The frequency-doubled laser beam was reflected from the interaction point on the sample onto the streak-camera photocathode, and recorded on the streak camera along with the UV timing fiducial referred to above. Thus the time of irradiation of the sample was known with respect to the UV fiducial. When the change in optical delay due to the insertion of optical components was taken into account, we estimate the accuracy of this absolute measurement of the timing to be $\pm 5 \mathrm{ps}$. After finding the absolute timing, the frequencydoubling crystal in the main beam was removed, and the window in front of the photocathode replaced with the beryllium one. A UV fiducial beam from the laser was recorded for every laser shot.

\section{4}

\section{Experimental results and simulations}

The X-ray-diffraction measurements were performed on asymmetrically cut InSb single-crystal wafers. The $\mathrm{X}$-rays were diffracted from the (111) planes, and the crystal was cut such that $\phi=7.2^{\circ}$. Initially, the X-ray energy was set at $8453 \mathrm{eV}$, corresponding to a Bragg angle, $\theta_{\mathrm{B}}$, of $11.3^{\circ}$. The grazing angle of incidence of the $\mathrm{X}$-rays with respect to the crystal surface was $\theta_{\mathrm{B}}-\phi=4.1^{\circ}$, and the angle of the exiting X-ray beam with respect to the crystal surface was $\theta_{\mathrm{B}}+$ $\phi=18.5^{\circ}$. When recording the rocking curves of the crystals, irradiated or otherwise, the X-ray energy was varied about its mean value by use of the double-crystal monochromator, with the angle of incidence, and hence diffraction, of the X-ray beam held fixed. This approach (rather than rocking the crys- tal) was used to ensure that the diffracted X-rays always fell on the streak-camera entrance slit. As the shifts in X-ray energy were relatively small, and the energy of the X-rays were far from any relevant absorption edges, such a shift in energy could readily be translated back to an effective angular shift, by a simple differentiation of Bragg's law. The maximum energy shifts employed, $\pm 35 \mathrm{eV}$, correspond to deviations in angle of $\pm 173 \operatorname{arcsec}( \pm 0.84 \mathrm{mrad})$.

As shown earlier in Sect. 2.2, the effective dispersion and bandwidth greatly influence the capability to observe the oscillations in the X-ray reflectivity corresponding to diffraction from the coherent phonons. The bandwidth and effective angular divergence of the beamline were checked by measuring the rocking curve of an unirradiated sample crystal, with asymmetry angle $\phi=-7.2^{\circ}$. Note that, for negative $\phi$, the theoretical rocking curve of the InSb is narrow (FWHM = $9.75 \operatorname{arcsec}=47 \mu \mathrm{rad})-$ and similar in magnitude to that of the computed rocking curve of the double-crystal monochromator (FWHM $\approx 7 \operatorname{arcsec} \approx 34 \mu \mathrm{rad}$ ), and thus in this geometry the measured reflectivity as a function of X-ray energy can yield an estimate of the effective bandwidth of the radiation from the monochromator. The observed reflectivity of the InSb as a function of energy, converted to angular units, is shown in Fig. 9a. The convolution of the theoretical reflection curves of the monochromator and the sample crystal is in good agreement with the measured rocking curve, confirming the calculated effective bandwidth of the monochromator. The vertical divergence angle (in the diffraction direction) of the X-ray beam is determined by the undulator, and is about $7 \mu \mathrm{rad}$, which is negligible.

The rocking curve of the InSb was also measured for the positive- $\phi$ geometry that was employed for the diffraction from coherent phonons. In this geometry the rocking-curve width of the InSb is considerably larger, and calculated to be a factor of 4.4 greater than the negative- $\phi$ case. Thus we would not expect the finite bandwidth due to the monochromator to greatly influence the measured width. As seen in Fig. 9b, in this case the calculated and measured rocking curves of the $\mathrm{InSb}$ are in very good agreement, even without convolving the monochromator rocking curve with that of the InSb.

Before irradiation of the InSb crystal, the temporal profile of the X-ray pulses from the electron bunches was measured by diffracting the X-rays from the surface of the InSb crystal 


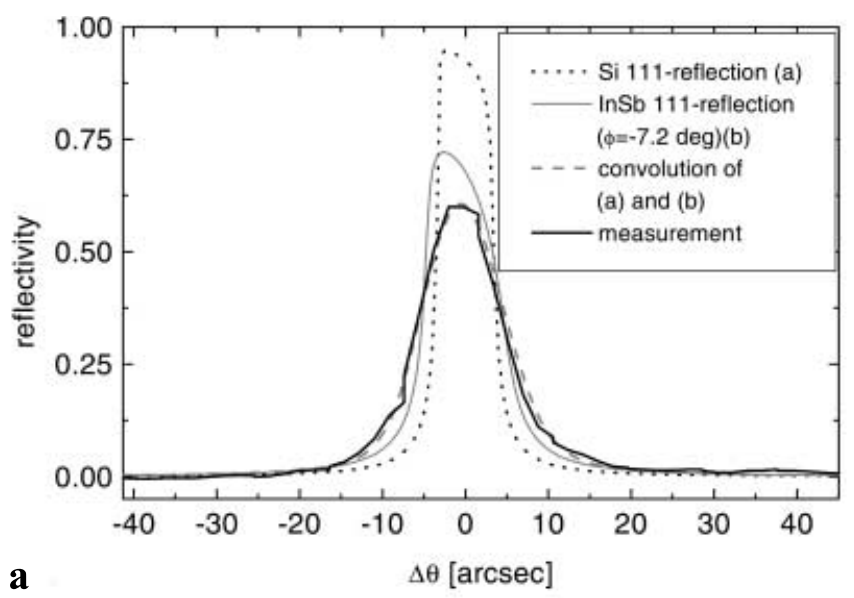

a

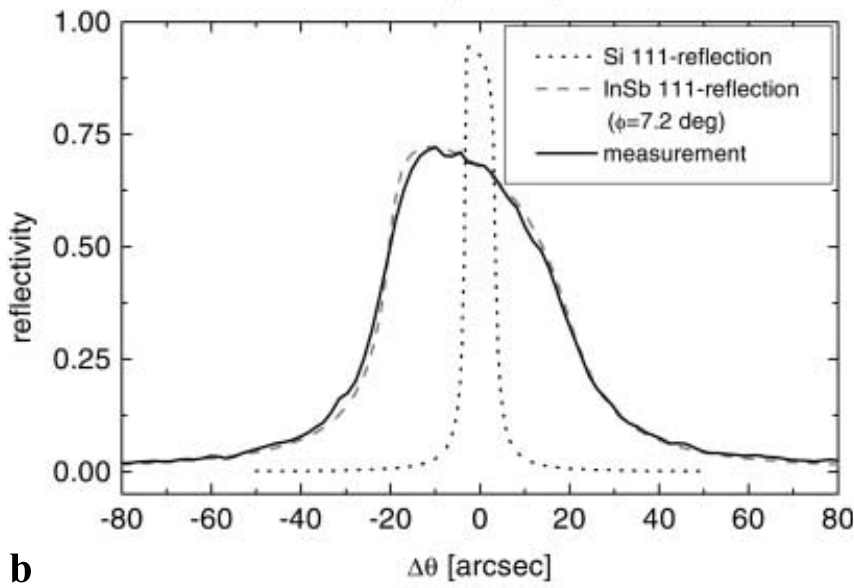

FIGURE 9 Calculated rocking curves of the Si monochromator and the InSb sample crystal together with the measured rocking curve of the sample crystal for $\mathbf{a}$ asymmetric InSb with $\phi=-7.2^{\circ}$ and $\mathbf{b}$ asymmetric InSb with $\phi=7.2^{\circ}$

and recording the result on the streak camera. The width of the pulse is found to be 85 ps (FWHM). The crystal was then irradiated with the laser. The angle between laser and X-ray beams was about $10^{\circ}$ and the angle between laser beam and target normal was of the order of $76^{\circ}$, which is the Brewster angle of InSb at a wavelength of $800 \mathrm{~nm}$. Thus the reflectivity of the crystal for the $p$-polarized laser radiation can be assumed to be close to zero, and the laser energy absorbed almost completely. The size of the laser focus on the sample was $2 \mathrm{~mm} \times 0.5 \mathrm{~mm}$, and was larger than the X-ray footprint on the sample. For each individual 100-fs laser pulse, the laser fluence was of the order of $1.5 \mathrm{~mJ} / \mathrm{cm}^{2}$. The millisecond interval between individual laser pulses is long compared with the time scale for diffusion into the sample, and for the fluences used here the crystal recovers between individual laser pulses, allowing accumulation of data over many, many laser shots.

The time-dependent reflectivity of the crystal was thus measured for a variety of incident X-ray energies, with the angle of incidence held fixed (though for ease of comparison with the simulations, we shall also plot this data on the equivalent angular scale).

The signal on the CCD camera coupled to the streak camera was integrated over a time ranging from 2.5 to $8 \mathrm{~min}$. During the integration time the time delay between the laser and the electron bunches was slowly and uniformly varied such that the leading edge of the X-ray pulse, as recorded on the streak camera, was registered at a position that varied from from $t=-50 \mathrm{ps}$ to $250 \mathrm{ps}$ relative to the time at which the laser irradiated the sample. For an exposure time of $7 \mathrm{~min}$ this corresponds to a 2-min integration time per 85-ps width (FWHM) of an X-ray pulse. The result of this procedure, as explained previously, is to produce a reflectivity measurement for a single scan that is longer than the intrinsic bunch length, without compromising the temporal resolution.

Figure 10a and $\mathrm{b}$ show the raw streak camera CCD images for $\Delta \theta=125 \operatorname{arcsec}$ and $\Delta \theta=-125$ arcsec, respectively (corresponding to keeping the angle of incidence fixed, and varying the incident energy by $\mathrm{eV}$ ). It can be seen that after irradiation by the laser (at time $t_{0}$ ) the X-ray reflectivity oscillates. However, the oscillations on the two sides of the rocking curve are not in phase: at $\Delta \theta=125$ arcsec the intensity of the diffracted beam rapidly drops after irradiation to about $30 \%$ of the value for the unperturbed crystal before returning to close to its former level. In contrast, for the case of $\Delta \theta=-125$ arcsec, the $\mathrm{X}$-ray signal initially rises after irradiation, with the peak reflectivities after irradiation being significantly higher than the unirradiated values. This behavior can be seen to be consistent with the simulations shown in Figs. 4-7, where after irradiation the reflectivity clearly drops on the positive-angle side of the rocking curve, but rises on the negative-angle side.

For more detailed analysis with simulations we produce lineouts from the raw CCD data, and these are averaged over the width of the X-ray signal along the streak-camera slit direction (i.e. perpendicular to the direction of temporal resolution). The signal is also background-corrected. For example Fig. 11 shows the diffracted X-ray intensity for an unperturbed crystal at the peak of the rocking curve and the much weaker X-ray intensities with laser on and off at a position +125 arcsec from the Bragg peak.

Data was collected over a wide range of incident energies around the mean energy, from $-25 \mathrm{eV}$ to $+35 \mathrm{eV}$, which cor-

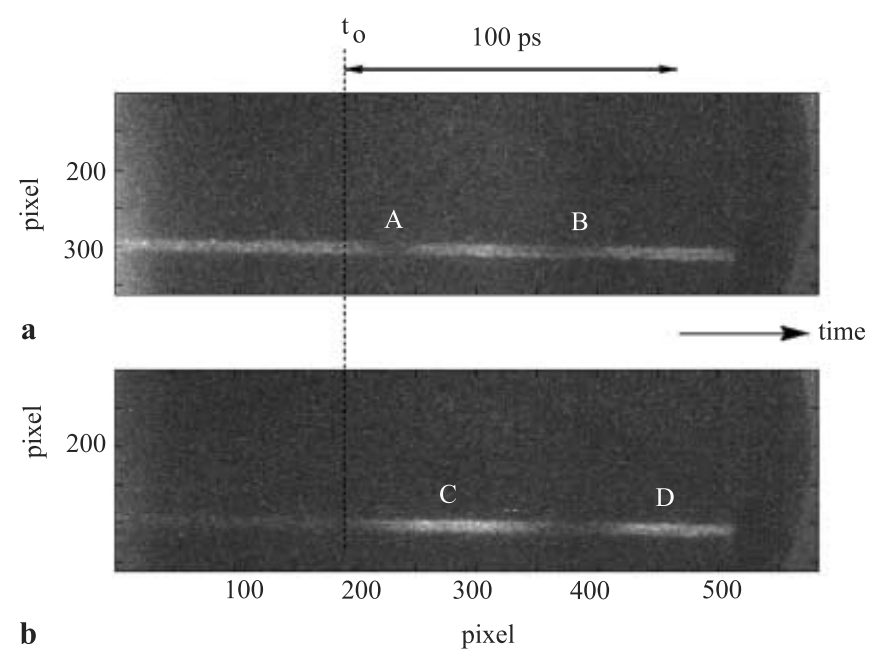

FIGURE 10 Raw streak-camera images from angular positions on both sides of the rocking curve. The laser hits the sample at time $t_{0}$. Recorded signals at a $\Delta \theta=125$ arcsec: the $\mathrm{X}$-ray signal drops almost to zero at positions $\mathrm{A}$ and $\mathrm{B} ; \mathbf{b} \Delta \theta=-125$ arcsec: the $\mathrm{X}$-ray signal rises to up to seven times the value of the unperturbed crystal at positions $\mathrm{C}$ and $\mathrm{D}$ 


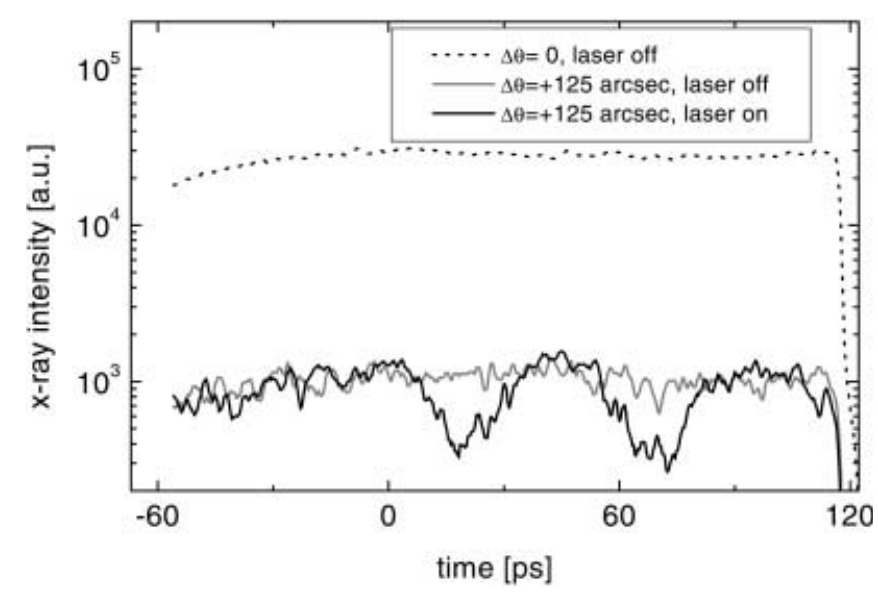

FIGURE 11 Time-dependent X-ray reflectivity at the Bragg peak of the unperturbed crystal, and at $\Delta \theta=125 \operatorname{arcsec}(0.6 \mathrm{mrad},+25 \mathrm{eV})$ with and without laser excitation of the sample crystal. Note that by use of the phase shifter the apparent duration of the X-ray pulse recorded on the streak camera is greater than the intrinsic bunch length

responds to angular shifts from -125 arcsec to +175 arcsec. The measured frequencies of the oscillations as a function of deviation from the Bragg angle were compared with the oscillation frequencies from the simulated reflectivity profiles. As can be seen in Fig. 12, the data and simulations are in very good agreement, and confirm the linear dependence between deviation from the Bragg angle and oscillation frequency. From a linear fit of the frequencies $\omega(\Delta \theta)$ deduced from the measured reflectivity profiles we find

$\Delta \omega / \Delta \theta=(194 \pm 10) \mathrm{THz} / \mathrm{rad}$

When combined with (2), we find that such a dispersion implies a sound speed of $v=(3750 \pm 200) \mathrm{m} / \mathrm{s}$, which is in good agreement with the tabulated velocity of longitudinal acoustic phonons in the [111] direction of $3880 \mathrm{~m} / \mathrm{s}$ (the deviation of the surface normal from the [111] direction is sufficiently small to have a negligible effect on the velocity of sound).

The frequency of the oscillations predicted by the simulations is relatively insensitive to the exact form of the strain

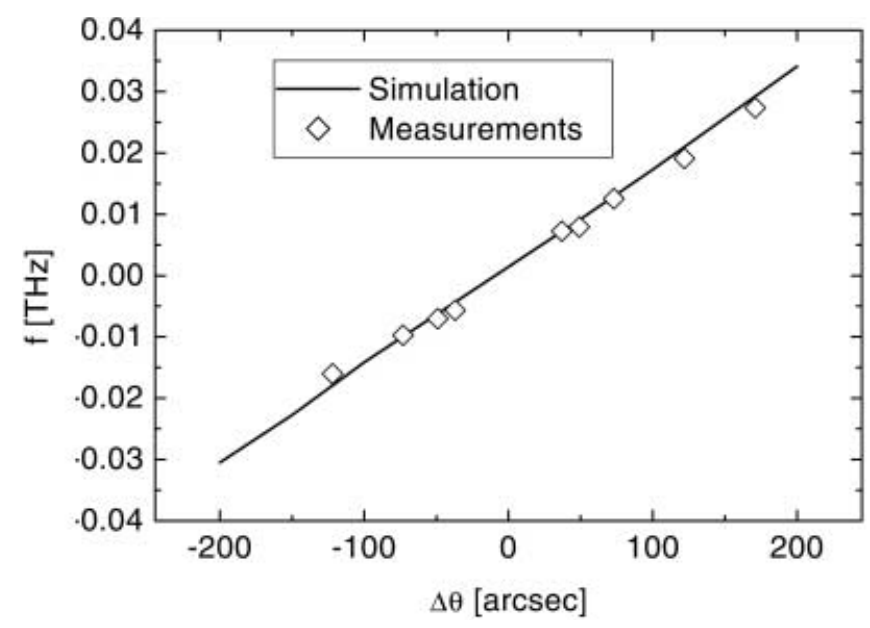

FIGURE 12 Oscillation frequencies from measurements and simulations profiles assumed to be propagating into the crystal (which is as to be expected, as a particular angular deviation from the original Bragg angle effectively picks out a particular phonon frequency). On the other hand the amplitude of the oscillations will be sensitive to the strain profile. If we assume the Thomsen model of strain propagation (i.e. the time-dependent strain profile evolving from an initially exponentially decaying stress profile), then in order to compare simulated diffraction profiles with those observed experimentally we need to know the absorption depth of the laser. Given the absorption depth, the maximum temperature reached at the crystal surface can be deduced (assuming that all of the laser energy goes into heating the crystal). The temperature at the crystal surface can also simply be related to the maximum strain produced at the surface, $\eta_{\max }$, once the crystal starts to relax. For a laser energy $Q$ per shot

$\eta_{\max }=\frac{Q \beta}{A \xi C \varrho} \frac{1+v}{1-v}$

where $A$ is the area of the laser focal spot, $\xi$ the laser penetration depth, $\beta=5.4 \times 10^{-6} / \mathrm{K}$ the linear thermal expansion coefficient, $C=144 \mathrm{~J} \mathrm{~kg}^{-1} \mathrm{~K}^{-1}$ the specific heat capacity, $\varrho=5.775 \mathrm{~g} / \mathrm{cm}^{3}$ the density and $v=0.2334$ the Poisson ratio for InSb in the [111] direction. The value $Q / A$ is the absorbed laser fluence assuming all laser energy goes into the thermal energy stressing and straining the crystal. This is a reasonable assumption, given that the band gap of $\operatorname{InSb}(0.17 \mathrm{eV})$ is small compared with the photon energy $(1.5 \mathrm{eV})$. From measurements of the focal spot size, we estimate the laser fluence, $Q / A$, to be 0.8 to $1.5 \mathrm{~mJ} / \mathrm{cm}^{2}$. Thus, assuming these fluences, we find a linear relation between absorption depth and peak strain at the crystal surface such that

$\eta_{\max } \xi=8-16 \mathrm{~nm}$

Given this constraint all of the measured time-dependent reflectivities for all of the observed angles are in agreement with the simulations assuming maximum strain in the range of $(0.05-0.15) \%$ and a laser penetration depth of $\xi=150 \mathrm{~nm}$. This can be seen with reference to Fig. 13 where the measured temporal profiles for 15 positions on the rocking curve are shown together with the simulation results. Two simulations with strains $0.05 \%$ and $0.1 \%$ are plotted together with the experimental results. The experimental curves show that the strain varies between $0.05 \%$ and $0.1 \%$. This can be seen from the fact that the experimental pulse shape is intermediate between the two simulations. The deviation in pulse form near center frequency can be compensated for by slightly shifting the absolute angle in the simulations. In the experiments such a shift of the relative angle in relation to the center position on the rocking curve can occur if the laser energy varies slightly over the data-collection time. This results in a slight change in the ambient temperature of the crystal, and a resultant change in mean lattice spacing and absolute position of the rocking curve.

It should be mentioned that any influence of a finite electron-phonon coupling time has been neglected within these simulations, yet reasonable agreement with the data is still seen. This is in contrast to the work of Lindenberg 

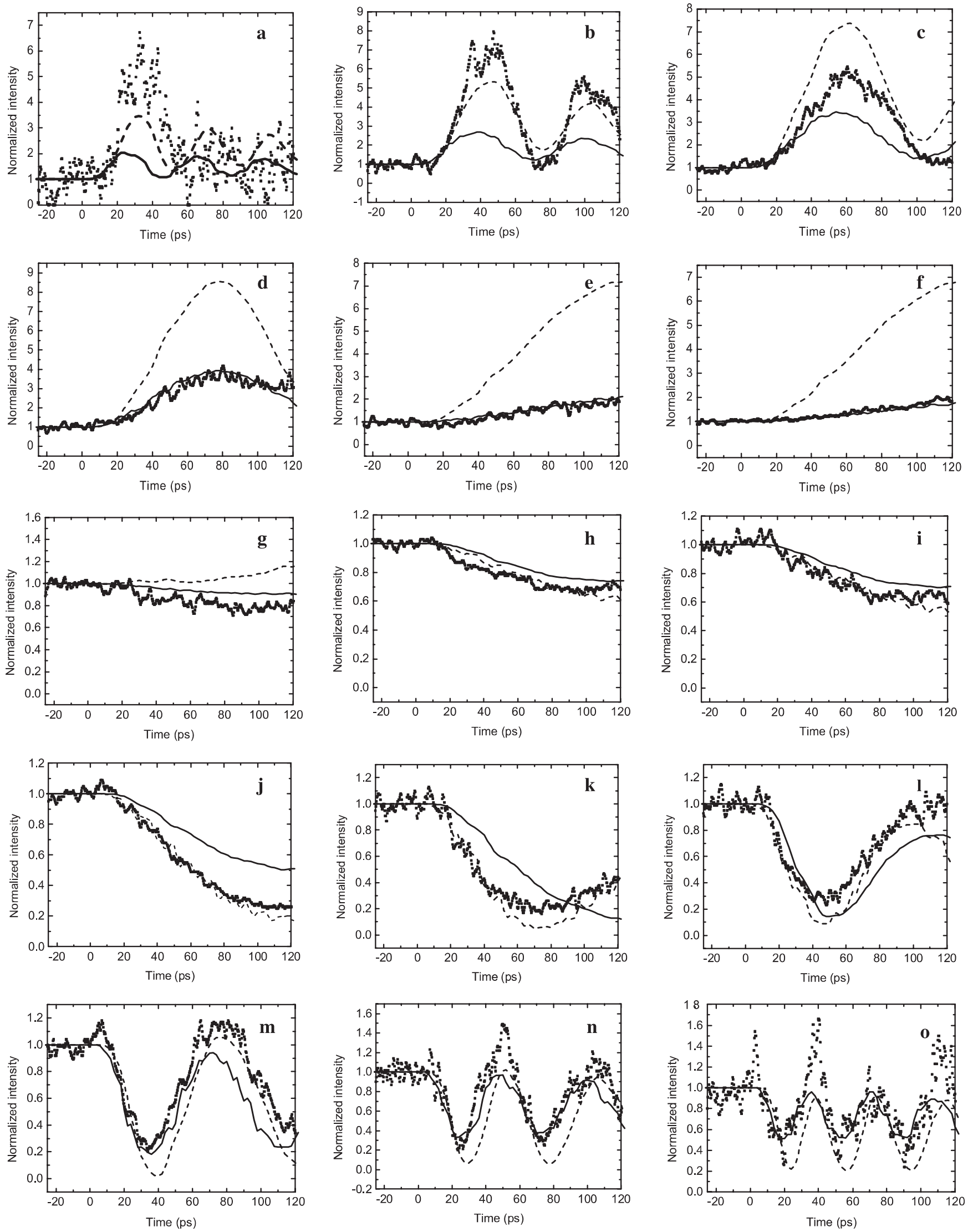

FIGURE 13 Measurements and simulations of the oscillations in the diffracted X-ray signal at 15 positions on the rocking curve. A laser penetration depth of $150 \mathrm{~nm}$ has been used for all simulations. For the simulations shown as solid lines, a peak strain of $0.05 \%$ has been used and for the dashed lines a strain of $0.10 \%$ has been used. The positions on the rocking curve are as follows $\mathbf{a}-175 \operatorname{arcsec}, \mathbf{b}-125 \operatorname{arcsec}, \mathbf{c}-75 \operatorname{arcsec}, \mathbf{d}-50 \operatorname{arcsec}, \mathbf{e}-37 \mathrm{arcsec}$, $\mathbf{f}-25 \operatorname{arcsec}, \mathbf{g}-12 \operatorname{arcsec} \mathbf{h} 0, \mathbf{i} 12 \operatorname{arcsec}, \mathbf{j} 25 \operatorname{arcsec}, \mathbf{k} 37 \operatorname{arcsec}, \mathbf{l} 50 \operatorname{arcsec}, \mathbf{m} 75 \operatorname{arcsec} \mathbf{n} 125 \operatorname{arcsec}, \mathbf{o} 175 \operatorname{arcsec}$ 
et al. [9], where they found that to get good agreement between simulated and observed time-dependent reflectivities over the whole data range they needed to assume a gradual, rather than infinitely sharp, interface between the regions of expansion and compression illustrated in Fig. 3. They attributed this finite strain gradient to the electron-phonon coupling time. This electron-phonon coupling has most effect on the time-dependent rocking curve for the oscillations with high frequency, with periods close to the coupling time itself. In [9], periods down to about 18 ps were interrogated (see Figs. 1 and 3 of [2]), for which the finite coupling time somewhat altered the simulated diffraction. However, in the work presented here, observations of coherent phonons were only made out to phonon periods of double this -35 ps or so (see Fig. 13). At this period the effect of the coupling time on the strain does not greatly alter the time-dependent diffraction. Evidently, given the above considerations, it would be of interest in the future to investigate which experimental setup would be best suited to observe finite electron-phonon effects in time-dependent X-ray diffraction. Furthermore, it is also clear from our data, and from a consideration of Figs. 4-7, that the low effective bandwidth of the source allows oscillations to be observed for longer than seen in [9]. From Fig. 5 we see that in [9] it was difficult to observe oscillations after about 50 ps (in agreement with Fig. 3 of [9]) due to finite-bandwidth effects, whereas in this work the observed oscillations in the $\mathrm{X}$-ray reflectivity still have large amplitudes $120 \mathrm{ps}$ after initiation of the phonon pulse (see Fig. 13).

We have investigated the dependence of the oscillation amplitude as the laser fluence is varied. We define the modulation as the difference between maximum and minimum oscillation amplitudes normalized to the initial reflectivity of the unperturbed crystal. We assume that the strain pulse form is not distorted with increasing fluence and by varying the laser fluence only the amplitude of the exponential strain profile changes. In Fig. 14, we plot the modulation as function of fluence.

The measurements indicate that the oscillation amplitudes on the expansion side of the reflection curve $(\Delta \theta<0)$ are more sensitive to the strain than those on the compression side. This is also seen in the simulations. Finally, the measurements confirm the approximately proportional dependence of the oscillation amplitude from the magnitude of the strain.

After having studied the deposition of energy into the material, we now discuss the dissipation of energy through heat conduction. Thomsen et al. [12] have found that the effect of heat conduction on the strain pulse traveling into the crystal material is determined by the value of $D / \xi v$, where $\xi$ denotes the penetration depth and $v$ the sound speed. For InSb, the diffusion coefficient $D$ is $0.16 \mathrm{~cm}^{2} / \mathrm{s}=16 \mathrm{~nm}^{2} / \mathrm{ps}$, the sound speed for longitudinal acoustic phonons in the [111] direction is $3.8 \mathrm{~nm} / \mathrm{ps}$ and the laser penetration depth is $150 \mathrm{~nm}$. These values lead to $D / \xi v=0.03$ and indicate only a small effect of heat conduction on the strain pulse, which would only have an influence on higher-frequency phonons not probed in this experiment. In Thomsen et al. [12] it is shown that the main features of the pulse remain intact as this value is as high as 1 . However, a distortion of some of the rapid features in the pulse shape is found already at a value of 0.1 .

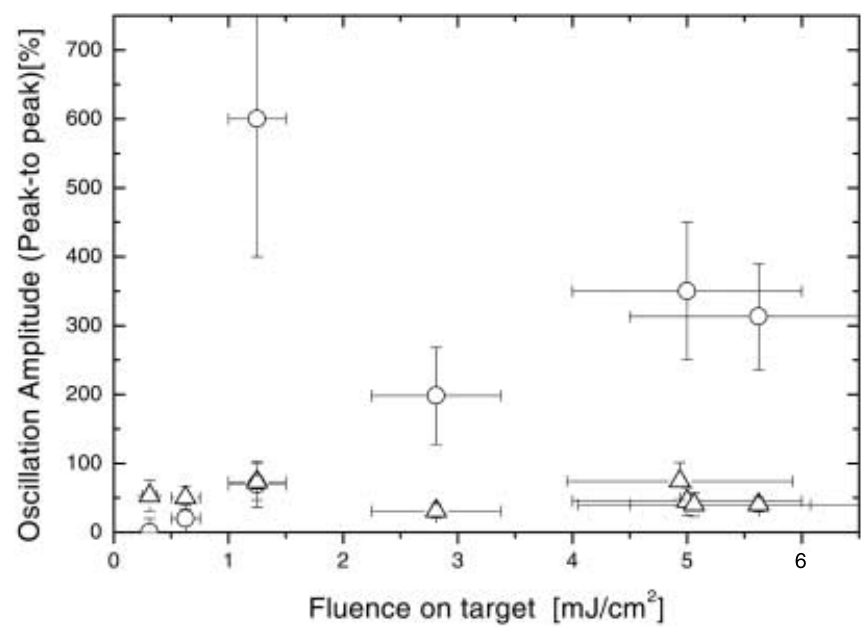

FIGURE 14 Modulations on the compression side of the rocking curve at $\Delta \theta=-130 \operatorname{arcsec}(\Delta E=+25 \mathrm{eV})$ (circles) and on the expansion side at $\Delta \theta=-130 \operatorname{arcsec}(\Delta E=+25 \mathrm{eV})$ (triangles) at as a function of maximum strain. The crosses indicate the error in the determination of modulation and fluence

In order to follow the strain propagation on a longer time scale, pump-probe measurements were performed just using the temporal resolution provided by the synchrotron X-ray pulses, i.e. leaving out the streak camera. The laser fluence on the target was about 12 to $16 \mathrm{~mJ} / \mathrm{cm}^{2}$, close to the damage threshold of the InSb sample crystal.

The recorded rocking curves for different times $t$ after the laser excites the crystal show that, on time scales of nanoseconds, the effects of heat conduction on the shape of the rocking curve cannot be ignored any more (Fig. 15). The intensity of the reflected signal has been measured with a Si diode and recorded over the deviation $\Delta \theta$ from the Bragg angle by rotating the sample crystal. The temporal resolution of this measurement is about $85 \mathrm{ps}$, corresponding to the duration (FWHM) of an X-ray pulse. At $t=2 \mathrm{~ns}$ the broadening of the rocking curve due to the variation of surface strain can clearly be seen. The rocking curve is not only broadened but also shifted to smaller angles due to the average crystal expansion over the probing depth. For later times the profile of the sur-

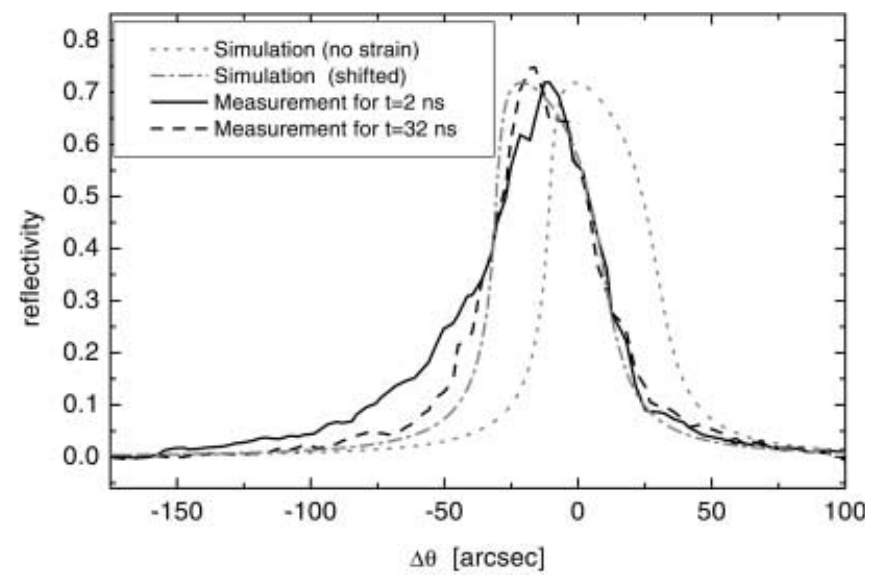

FIGURE 15 Rocking curves of $\operatorname{InSb}(111)$ at $t=2 \mathrm{~ns}$ and $t=32 \mathrm{~ns}$ after the laser pulse maximum. Also shown: calculated reflection curves of the unperturbed crystal and the same curve shifted by -20 arcsec, corresponding to a homogeneous strain 
face strain flattens out more and more due to heat conduction. The rocking curve at $t=32 \mathrm{~ns}$, shown in Fig. 15, is similar to the reflection curve of the unperturbed crystal. It is only shifted by 20 arcsec towards smaller angles due to an increase in average temperature by $\Delta T=11 \mathrm{~K}$.

In simulations, an analytic solution for the time-dependent strain close to the surface has been used to calculate the timedependent reflection curve. The results of the simulation are in very good agreement with the measured rocking curve. This shows that time-resolved X-ray Bragg reflection is a very sensitive and useful tool to monitor the evolution of strain in crystals.

\section{$5 \quad$ Conclusion}

In the extensive set of experimental data and simulations presented here we have performed a detailed investigation of coherent longitudinal acoustic phonons in InSb using time-resolved X-ray diffraction. Coherent acoustic phonons lead to large-amplitude oscillations of the Bragg-diffracted $\mathrm{X}$-ray intensity on both sides of the rocking curve, which have been detected in the present experiment. These oscillations have the same frequency as the acoustic phonons they are originating from. This frequency depends on the Bragg angle mismatch $\Delta \theta$ at which the oscillations are recorded.

It has been proven theoretically and experimentally that the angular dispersion $\Delta \omega / \Delta \theta$ of the diffracting sample crystal can be enhanced by using asymmetrically cut sample crystals in a grazing incidence Bragg-diffraction configuration. This allows a higher angular resolution and therefore a longer time period over which oscillations can be observed. This is due to the fact that the dephasing effect due to frequency differences of oscillations from neighboring points on the rocking curve is reduced simply because the rocking curve for monochromatic radiation is convolved with an instrumental function of narrower fractional effective bandwidth. This has allowed us, in contrast to previous measurements reported by other groups [2,9], to observe large-amplitude oscillations in the X-ray reflectivity for longer than 120 ps. Further measurements have to be performed to investigate the temporal behavior of acoustic phonons on a longer time scale. The excitation process of the acoustic phonons within the first few picoseconds has to be studied at higher frequencies further off the center of the rocking curve.

In the present experiment phonons could be detected for a lower laser fluence than in previous studies. In accordance with the strain model, the amplitude of the observed oscillations in this low-fluence regime scales linearly with the fluence.

ACKNOWLEDGEMENTS J.L. and T.M. would like to thank the Swedish Natural Science Research Foundation (NFR). The authors would like to acknowledge the financial contribution of the European Commission through the Human Potential Programme.

\section{REFERENCES}

1 C. Rischel, A. Rousse, I. Uschmann, P.-A. Albouy, J.-P. Geindre, P. Audebert, J.C. Gauthier, E. Förster, J.-L. Martin, A. Antonetti: Nature 390, 480 (1997)

2 C. Rose-Petruck, R. Jimenez, T. Guo, A. Cavalleri, C.W. Siders, F. Raksi, J.A. Squier, B.C. Walker, K.R. Wilson, C.P.J. Barty: Nature 398, 310 (1999)

3 R.W. Schoenlein, S. Chattopadhyay, H.H.W. Chong, T.E. Glover, P.A. Heimann, C.V. Shank, A.A. Zholent, M.S. Zolotorev: Science 287, 2237 (2000)

4 H. Maris: Sci. Am. Int. Ed. 278, 64 (1998)

5 S. Takagi: Acta Crystallogr. 15, 1311 (1962)

6 J. Burgeat, D. Taupin: Acta Crystallogr. A 24, 99 (1968)

7 C.R. Wie, T.A. Tombrello, T. Vreeland, Jr.: J. Appl. Phys. 59, 3743 (1986)

8 J.S. Wark, R.W. Lee: J. Appl. Crystallogr. 32, 692 (1999)

9 A.M. Lindenberg, I. Kang, S.L. Johnson, T. Missalla, P.A. Heimann, Z. Chang, J. Larsson, P.H. Bucksbaum, H.C. Kapteyn, H.A. Padmore, R.W. Lee, J.S. Wark, R.W. Falcone: Phys. Rev. Lett. 84, 111 (2000)

10 D.A. Reis, M. DeCamp, P.H. Bucksbaum, R. Clarke, E. Dufresne, M. Hertlein, R. Merlin, R. Falcone, H. Kapteyn, M. Murnane, J. Larsson, T. Missalla, J. Wark: Phys. Rev. Lett. 86, 3072 (2001)

11 M.F. DeCamp, D.A. Reis, P.H. Bucksbaum, B. Adams, J.M. Caraher, R. Clarke, C.W.S. Conove, E.M. Dufresne, R. Merlin, V. Stoica, J.K. Wahlstrand: Nature 413, 827 (2001)

12 C. Thomsen, H.T. Grahn, H.J. Maris, J. Tauc: Phys. Rev. B 34, 4129 (1986)

13 D. Bourgeois, T. Ursby, M. Wulff, C. Pradervand, A. Legrand, W. Schildkamp, S. Labourand, V. Srajer, T.Y. Teng, M. Roth, K. Moffat: J. Synchrotron Radiat. 3, 65 (1996)

14 J. Larsson, Z. Chang, E. Judd, P. Schuck, R. Falcone, P. Heimann, H.A. Padmore, H.C. Kapteyn, P. Bucksbaum, M. Murnane, R.W. Lee, A. Machachek, J. Wark: Opt. Lett. 22, 1012 (1997)

15 K. Scheidt, G.A. Naylor: In Proc. 4th Eur. Workshop Beam Diagn. Instrum. Part. Accel., Chester, UK, May 16-18, 1999, p. 51 [also available at http: //accelerator.dl.ac.uk/DIPAC/proceedings.pdf, p. 60] 